
3 Research Square
Preprints are preliminary reports that have not undergone peer review.
They should not be considered conclusive, used to inform clinical practice,
or referenced by the media as validated information.

\title{
Mycobacterium mucogenicum and Mycobacterium neoaurum Bloodstream Infection in Immunocompromised Hosts
}

Armelle Pérez-Cortés Villalobos

University of Toronto

Coleman Rotstein ( $\nabla$ coleman.rotstein@uhn.ca)

University Health Network https://orcid.org/0000-0003-1413-0968

Research article

Keywords: Nontuberculous mycobacteria, bacteremia, infection, mycobacteria

Posted Date: May 29th, 2020

DOI: https://doi.org/10.21203/rs.3.rs-29851/v1

License: (9) (7) This work is licensed under a Creative Commons Attribution 4.0 International License. Read Full License 


\section{Abstract \\ Background}

Mycobacterium mucogenicum and Mycobacterium neoaurum are an infrequent cause of bloodstream infection in humans. When they are isolated from blood, it is not unusual for them to be considered to be contaminants initially.

\section{Methods}

A retrospective chart review of patients diagnosed with M. mucogenicum and M. neoaurum bacteremia was conducted from January 1998 to December 2018 at the University Health Network, Toronto, Canada.

\section{Results}

We identified 28 patients who had bloodstream infection caused by these organisms. These patients were immunocompromised hosts, patients with chronically indwelling vascular access devices or injection drug users. In $28.5 \%$ of the patients the isolation of these mycobacteria was considered colonization, $82 \%$ of the cases received antimicrobial therapy and in $95 \%$ of the patients the central line was removed.

\section{Conclusions}

The removal of the vascular access device and treatment with either single or combination antimicrobial therapy produced successful outcomes for these infections.

\section{Background}

With the expansion of the immunocompromised patient population, infections due to nontuberculous mycobacteria (NTM) are increasing in frequency ${ }^{1}$, and their impact is significant as they may produce considerable patient morbidity. As a result, more familiarity with these organisms and the infections that they may produce is required. There are currently more than 150 species of NTM that are divided into rapidly, intermediate and slowly growing species ${ }^{2}$.

Rapidly growing mycobacteria produce mature colonies on solid media within 7 days. There are about 20 species of rapidly growing NTM that are capable of infecting human beings ${ }^{3}$. Of interest among the rapidly growing NTM, are two less commonly isolated mycobacteria, Mycobacterium mucogenicum and Mycobacterium neoaurum. They are ubiquitous in the environment, including household water, potting soil, animals, birds and vegetables. These organisms have been increasingly noted to be the cause of significant infections in immunocompromised hosts.

The current literature highlights that the most common infection caused by $M$. mucogenicum and $M$. neoarum is catheter-related bloodstream infection $(\mathrm{CRBSI})^{4-7}$. The frequent use of indwelling intravascular catheters in immunocompromised individuals has been accompanied by NTM CRBSI, especially in patients with underlying malignancies ${ }^{9}$.

These organisms have the ability to form a protective biofilm, which plays a role in CRBSI. Biofilms in turn confer decreased penetration to antimicrobials, enhance the potential of resistance and are integral to device-related infections ${ }^{9}$. These organisms are also able to tolerate disinfectants, chlorination and extreme temperatures.

M. mucogenicum and M. neoaurum infections are seen more often in long-term central intravenous catheters causing catheter-related sepsis, but they may also occur with peritoneal or shunt catheters. Central nervous system infections involving Mycobacterium mucogenicum are rare but serious, particularly for immunocompromised patients resulting in meningitis due to this organism ${ }^{5}$. Skin and soft tissue infections caused by these organisms have also been reported ${ }^{4,10}$

In immunocompromised patients, M. mucogenicum or M. neoaurum isolated from the bloodstream should be considered as true pathogens. The treatment of these NTM CRBSI involves catheter removal combined with antibiotic therapy ${ }^{3}$. These species are usually susceptible to multiple antimicrobial agents including aminoglycosides, cefoxitin, clarithromycin, minocycline, doxycycline, quinolones, trimethoprim/sulfamethoxazole, and imipenem ${ }^{11}$. Currently, there is no guidance or consensus on the appropriate antimicrobial therapy (whether single agent or combination therapy) for these infections or the duration of treatment for infections caused by $\mathrm{NTM}^{8}$.

Further information is needed to better understand the epidemiology including predisposing host factors, antibiotic susceptibility patterns, and appropriate duration of therapy for these infections particularly bloodstream infections caused by M. mucogenicum and M. neoaurum.

\section{Methods}

A retrospective chart review of patients diagnosed with M. mucogenicum and M. neoaurum bacteremia was conducted from January 1998 to December 2018 at the Toronto General Hospital and Princess Margaret Cancer Centre of the University Health Network, Toronto, Ontario, Canada. This project was approved by the Research Ethics Board of the University Health Network. 
We identified patients with infection due to M. mucogenicum and M. neoaurum cultures through a search of the Microbiology Laboratory database of the Mount Sinai Hospital. The microbiological isolates were confirmed with Geno Type Mycobacterium Assay for identification of Mycobacterial Species or by matrix assisted laser desorption ionization (MALDI-TOF) if available. Only two isolates of $M$. mucogenicum had in vitro susceptibility testing, that was done by a microdilution minimum inhibitory concentration (MIC) method. Susceptibility testing for the mycobacterial isolates was performed at the Public Health Ontario Laboratories. We excluded patients who were younger than 18 years old or whose source of the culture was other than the bloodstream.

Data collection included patient demographics, comorbidities, major underlying predisposing condition, origin of the positive blood culture for $M$. mucogenicum and $M$. neoaurum, microorganism susceptibility patterns if available, presence and site of intravascular catheters, clinical manifestations, treatment regimen and duration, as well as outcome.

We used the Centers for Disease Control definition of central line-associated bloodstream infection. ${ }^{12}$ This was defined as a patient who had at least one of the following signs or symptoms: fever $\left(>38^{\circ} \mathrm{C}\right)$, chills or hypotension, had a microorganism identified in blood not related to an infection at another site and whose microorganism was identified by culture or non-culture based microbiologic testing method, from two or more blood specimens collected on separate occasions. We employed the differential time to positivity i.e. the growth of organism from blood samples drawn from a catheter hub at least 2 hours before microbial growth is detected in a blood sample obtained from a peripheral vein, in order to define $\mathrm{CRBSI}^{13}$. Catheter-related infection was also determined by the presence of $\geq 15$ colony forming units on the vascular access device tip according to the semiquantitative method of Maki ${ }^{14}$.

Descriptive statistics were employed as appropriate to compare patients with successful outcomes to those with unfavourable outcomes.

\section{Results}

Between January 1998 to December 2018, we identified 81 isolates of M. mucogenicum, 24 of which were blood culture isolates, 3 from dialysis fluid, and 54 respiratory isolates from bronchoalveolar lavage fluid or sputum. M. mucogenicum was not considered to be a significant pathogen if the origin was the respiratory tract. We also identified 4 blood culture isolates of $M$. neoaurum. Therefore, we assessed a total of 28 episodes of bacteremia.

Of the 24 patients who developed M. mucogenicum bacteraemia, $15 / 24$ (62.5\%) were male, with a mean age was 53.3 years (21-87 years). Of the 4 patients who developed M. neoaurum bacteremia, 3/4 (75\%) were male and the mean age was 52.5 years (24-78 years).

The patient characteristics are shown in Table 1.

Table 1

Patient demographics

\begin{tabular}{|c|c|c|}
\hline & Mycobacterium mucogenicum & Mycobacterium neoaurum \\
\hline & Bacteremia $n=24$ & Bacteremia $n=4$ \\
\hline Male & $15(62.5 \%)$ & $3(75 \%)$ \\
\hline Age & Mean 53.3 (21-78 years) & Mean 52.2 (24-78 years) \\
\hline \multirow[t]{11}{*}{ Underlying disease } & 13 Hematological malignancy & 3 Hematological malignancy \\
\hline & 6 (46\%) Allogenic HSCT & 2 (66.6\%) Allogenic HSCT \\
\hline & $4(30 \%)$ Acute leukemia & 1 Solid tumor \\
\hline & 1 (8\%) Autologous HSCT & \\
\hline & $1(8 \%)$ Multiple myeloma & \\
\hline & 1 (8\%) Lymphoma & \\
\hline & 5 IV Drug users & \\
\hline & 3 Autoimmune diseases & \\
\hline & 1 Kidney transplant recipient & \\
\hline & 1 Solid tumor & \\
\hline & 1 Chronic kidney disease on hemodialysis & \\
\hline Steroid use & $18(75 \%)$ & $4(100 \%)$ \\
\hline
\end{tabular}

The most frequent comorbidity noted in the patients was hematological malignancies (Table 1). Twenty-five per cent of the patients had a history of allogeneic stem cell transplant in the $M$. mucogenicum bacteremia group and $50 \%$ in the $M$. neoaurum group. Most of the patients were receiving corticosteroids: $75 \%$ in the M. mucogenicum group and $100 \%$ in the $M$. neoaurum group. In $71.4 \%(20 / 28)$ of the bacteremias reviewed, a central venous access device was in place.

While $68 \%$ of the patients were lymphocytopenic, only 4 (14\%) were neutropenic at the time of the bacteremia (Table 2). Concomitant bloodstream infection with another pathogen was very common in the M. neoaurum group (75\%) two patients had concomitant infection with methicillin-susceptible

Page 3/7 
Staphylococcus aureus (MSSA) and one with Micrococcus luteus. Of note, the time to positivity of the blood culture for both M. mucogenicum and M. neoaurum was consistently greater than 24 hours.

Table 2

Clinical characteristics

\begin{tabular}{|c|c|c|}
\hline & $\begin{array}{l}\text { Mycobacterium mucogenicum } \\
\text { Blood culture } \mathrm{n}=24\end{array}$ & $\begin{array}{l}\text { Mycobacterium neoaurum } \\
\text { Blood culture } \mathrm{n}=4\end{array}$ \\
\hline Fever & $20(83 \%)$ & $2(50 \%)$ \\
\hline Hemodynamical instability & 0 & 0 \\
\hline $\begin{array}{l}\text { Hemoglobin } \\
(\mathrm{g} / \mathrm{dL})\end{array}$ & $103.8(66-146)$ & $96.5(75-113)$ \\
\hline $\begin{array}{l}\text { White count } \\
\text { (x10 E9/L) }\end{array}$ & $6.08(0.1-11.5)$ & $8.3(2.7-17.7)$ \\
\hline $\begin{array}{l}\text { Neutropenia (patients) } \\
(<0.5 \times 10 \mathrm{E} 9 / \mathrm{L})\end{array}$ & $4(16 \%)$ & 0 \\
\hline $\begin{array}{l}\text { Patients with lymphopenia } \\
(\leq 1 \times 10 \mathrm{E} 9 / \mathrm{L})\end{array}$ & $15(62.5 \%)$ & $4(100 \%)$ \\
\hline Platelet count (x10e9/L) & $141(6-474)$ & $144(99-175)$ \\
\hline Coinfection with another microorganism & 0 & $3(75 \%)$ \\
\hline Central line present & $16(66.6 \%)$ & $4(100 \%)$ \\
\hline Mean time to culture positivity (Hours) & $35.58(3-120)$ & $24.5(8-36)$ \\
\hline Number of positive blood cultures & $16(67 \%)$ & $2(50 \%)$ \\
\hline One & $3(12 \%)$ & $2(50 \%)$ \\
\hline Two & $2(9 \%)$ & \\
\hline Three & $3(12 \%)$ & \\
\hline Four & & \\
\hline
\end{tabular}

With regards to management, central venous access device removal ensued in $95 \%(23 / 24)$ of the $M$. mucogenicum infection and $75 \%$ with $M$. neoaurum However, none of the tip cultures were positive. Antimicrobial therapy was administered in 23 of the 28 (82\%) episodes of bacteremia. The antibiotic susceptibilities of the $2 \mathrm{M}$. mucogenicum isolates are demonstrated in Table 3 . Therapy with a single antimicrobial agent was undertaken in only $35 \%$ (8/23); while combination therapy was initiated in $65 \%$ (15/23 episodes). In addition, moxifloxacin was part of the combination regimen in all 15 courses of combination therapy. Most commonly, moxifloxacin was paired with amikacin and/or a carbapenem in 6 episodes (40\%) each respectively (Table 4).

Table 3

Microbiological Susceptibility of 2 Isolates of M. mucogenicum

\begin{tabular}{|c|c|c|c|c|c|c|c|c|c|c|}
\hline $\begin{array}{l}\text { Mycobacterium } \\
\text { mucogenicum }\end{array}$ & Amikacin & Cefoxitin & Ciprofloxacin & Clarithromycin & Doxycycline & Imipenem & Linezolid & Moxifloxacin & Tobramycin & Sep1 \\
\hline Isolate 1 & $S$ & $S$ & $S$ & S & $S$ & S & $S$ & S & $S$ & $S$ \\
\hline Isolate 2 & $S$ & I & $S$ & $S$ & $S$ & $S$ & $\mathbf{R}$ & I & $S$ & $S$ \\
\hline
\end{tabular}


Table 4

Outcomes

\begin{tabular}{|c|c|c|}
\hline & $\begin{array}{l}\text { Mycobacterium mucogenicum } \\
\mathrm{n}=24\end{array}$ & $\begin{array}{l}\text { Mycobacterium neoaurum } \\
\mathrm{n}=4\end{array}$ \\
\hline Considered colonization & $8(28.5 \%)$ & $1(25 \%)$ \\
\hline Received antimicrobial treatment & $20(83 \%)$ & $3(75 \%)$ \\
\hline Single agent & $8 / 20(40 \%)$ & 0 \\
\hline Combination therapy & $12 / 20(60 \%)$ & $3(100 \%)$ \\
\hline Single agent & $8(40 \%)$ & $3(100 \%)$ \\
\hline Ciprofloxacin or Moxifloxacin & $2(25 \%)$ & $1(33.3 \%)$ \\
\hline Ceftazidime or Ceftriaxone & $3(38 \%)$ & $1(33.3 \%)$ \\
\hline Meropenem & $1(12 \%)$ & $1(33.3 \%)$ \\
\hline Piperacilin/Tazobactam & $2(25 \%)$ & \\
\hline Combinations used & $12(60 \%)$ & \\
\hline Moxifloxacin/Pipercillin tazobactam & $3(25 \%)$ & \\
\hline Moxifloxacin/ Doxycycline & 0 & \\
\hline Moxifloxacin/Azithromycin & $1(8 \%)$ & \\
\hline Moxifloxacin/Imipenem & $3(25 \%)$ & \\
\hline Moxifloxacin/Amikacin or tobramycin & $2(17 \%)$ & \\
\hline Moxifloxacin/TMP-SMX & $1(8 \%)$ & \\
\hline Moxifloxacin/Carbapenem/Amikacin & $2(17 \%)$ & \\
\hline \multicolumn{3}{|l|}{ Moxifloxacin/Doxycycline/Amikacin } \\
\hline Duration therapy (days) & $20.6(7-42)$ & $22.5(12-34)$ \\
\hline Line removal & 16/16 (100\%) & $3 / 4(75 \%)$ \\
\hline Positive catheter tip & 0 & 0 \\
\hline 30 day survival & $100 \%$ & $100 \%$ \\
\hline 1-year survival & 21 (87.5\%) & $3(75 \%)$ \\
\hline
\end{tabular}

The outcomes for these cases of bacteremia were varied (Table 4). In 9 (32\%) patients, the organisms were initially considered colonization/contaminant. In the M. mucogenicum group, the organism isolated was initially considered to be a contaminant in 4 patients, but all of them had repeated blood cultures that were also positive and were treated. In addition, 3 patients with positive blood cultures were discharged and there were no available records to assess whether the infection was ever treated. In one patient, the first blood culture was considered a contaminant and because the repeat blood cultures were negative no treatment was provided. In the $M$. neoaurum group, only one patient did not receive treatment, as he had coinfection with methicillin-susceptible Staphylococcus aureus (MSSA) and only received therapy with cefazolin.

None of the patients had disseminated disease or another infection site caused by the mycobacterial infection. The 30 -day survival was $100 \%$ in both groups and one-year survival was $87.5 \%$ in the M. mucogenicum and $75 \%$ in the $M$. neoaurum $75 \%$ groups, respectively.

\section{Discussion}

This case series highlights that $M$. mucogenicum and $M$. neoaurum may produce significant infection in immunocompromised hosts. Commonly, these pathogens produce morbidity by causing CRBSI in this population.

Previous reports of bacteremia caused by these rapidly growing mycobacteria have focused in immunocompromised hosts. However, in this case series we found that all the immunocompetent patients, had an intravascular indwelling catheter at the time of diagnosis, this association has also been reported in the past ${ }^{16}$. We also found that 5 patients were chronic injection drug users. Therefore, the use of intravenous drugs should be recognized as a risk factor for the development of infections caused by these organisms.

Of note lymphocytopenia, was present in $68 \%$ of our patients, rather than neutropenia and may have predisposed our patients to these infections. Furthermore, these organisms have a predilection for causing CRBSI as was seen in our series ( $68 \%$ of the patients). Of significance, M. mucogenicum can form a biofilm on venous access devices thus rendering it resistant to killing by antimicrobials. ${ }^{3,6}$ As a result, it is strongly advised that vascular access devices be removed. In this case series the central line was removed in $95 \%$ of the patients, very similar to what has been reported in other studies ( $96 \%)^{3}$. 
With regard to the management of these bloodstream infections, in our series 5 of 28 episodes (18\%) were not treated with antimicrobial therapy. Indeed, the isolation of these organisms in blood cultures may be somewhat delayed further casting doubt on their significance and delaying appropriate antimicrobial therapy. A testament to this issue was that It was surprising that 5 patients didn't receive any antimicrobial therapy with purported activity against $M$. mucogenicum in our case series but they recovered by means of venous access device removal. Thus, underscoring the need for removal of the venous access as a key component for successful treatment of these bacteremias. Combination antimicrobial therapy involving moxifloxacin with amikacin or a carbapenem may be preferred. Sixty-five per cent of the patients in our series received a combination of antimicrobial agents involving moxifloxacin with another agent. Nevertheless, one active antimicrobial agent may be adequate. This would require verification in a randomized clinical trial.

These organisms have often been considered to be contaminants. Indeed, their clinical significance was not appreciated until persistent bacteremia was noted with resolution of the infection only after venous access removal with or without antimicrobial therapy as noted in 9 cases in our series. It should be noted that co-infections with Staphylococcus aureus may occur with M. neoaurum as noted in 2 of our patients.

As this was a retrospective review, our analysis was limited by the availability of the data. Thus, we did encounter missing information. Moreover, management of these bacteremias was also hampered by the lack antimicrobial susceptibility in all isolates. However, the data do underscore the morbidity caused by M. mucogenicum and M. neoaurum in immunocompromised hosts.

\section{Conclusions}

In summary, this report focuses on the morbidity of bacteremia caused by M. mucogenicum and M. neoaurum in immunocompromised patients that is often related to venous access devices and use of IV drugs. It highlights the need for venous access device removal and therapy with antibiotics that should include moxifloxacin.

\section{Abbreviations}

- Nontuberculous mycobacteria (NTM)

- Catheter-related bloodstream infection (CRBSI)

- Matrix assisted laser desorption ionization (MALDI-TOF)

- Minimum inhibitory concentration (MIC)

- Methicillin-susceptible Staphylococcus aureus (MSSA)

\section{Declarations}

\section{ETHICS APPROVAL}

This project was approved by the Research Ethics Board of the University Health Network, Toronto, Canada.

\section{CONSENT FOR PUBLICATION}

Not applicable. This manuscript does not contain any individual person's data in any form.

\section{AVAILABILITY OF DATA AND MATERIALS}

The datasets used and/or analysed in the current study are available from the corresponding author on request.

\section{COMPETING INTERESTS}

Dr. Coleman Rotstein is a member of the editorial board of Biomed Central Infectious Diseases (Associate editor).

\section{FUNDING}

There was no funding provided for this project.

\section{AUTHORS CONTRIBUTIONS}

Conception and design: All authors

\section{ACKNOWLEDGMENTS}


None.

\section{References}

1. Henkle E. WinthropK. Nontuberculous mycobacteria infections in immunosuppressed hosts. Clin Chest Med. 2015;36:91-9.

2. Mandell. Douglas, and Bennett's Principles and Practice of Infectious Diseases. 9 ed. Philadelphia: PA Elsevier/ Saunders; 2019.

3. El Helou G, Viola GM, Hachem R, Han XY, Raad II. Rapidly growing mycobacterial bloodstream infections. Lancet Infect Dis. 2013;13:166-74.

4. Adékambi T. Mycobacterium mucogenicum group infections: a review. Clin Microbiol Infect. 2009;15:911-8.

5. Abidi MZ, Lededboer N, Banerjee A, Hari P, et al. Mycobacterium mucogenicum bacteremia in immune-compromised patients, 2008-2013. Diag Microbiol Infect Dis. 2016;85:182-5.

6. Pradier M, Boucher A, Robineau O, Chachaty E, Meybeck A, Senneville E. Mycobacterium mucogenicum bacteremia: major role of clinical microbiologists. BMC Infect Dis. 2018;18(1):646.

7. Washer LL, Riddell IVJ, Rider J, Chenoweth CE. Mycobacterium neoaurum bloodstream infection: Report of 4 cases and review of the literature. Clin Infect Dis. 2007;45:e10-3.

8. Wallace RJ, Swenson JM, Slicox VA, et al. Spectrum of disease due to rapidly growing mycobacteria. Rev Infect Dis. 1983;5:657-79.

9. Esteban J, Martin-de-Hijas NZ, Kinnari TJ, Ayala G, Fernandez-Roblas R, Gadea I. Biofilm development by potentially pathogenic non-pigmented rapidly growing mycobacteria. BMC Microbiol. 2008;8:184.

10. Kim C-K, Choi SI, Jeon BR, Lee Y-W, Yk L, Shin HB. Pulmonary infection caused by Mycobacterium neoaurum: The first case in Korea. Ann Lab Med. 2014;34:243-6.

11. Wallace RJ Jr, Silcox VA, Tsukamura M, et al. Clinical significance, biochemical features, and susceptibility patterns of sporadic isolates of the Mycobacterium chelonaelike organism. J Clin Microbiol. 1993;31:3231-9.

12. CDC. Central line-associated bloodstream infection and non-central line associated bloodstream infection. January 2019. https://www.cdc.gov/nhsn/pdfs/pscmanual/4psc_clabscurrent.pdf.

13. Mermel L, Allon M, Bouza E, et al. Clinical practice guidelines for the diagnosis and management of intravascular catheter-related infection: 2009 Update by the Infectious Diseases Society of America. Clin Inf Dis. 2009;49:1-45.

14. Maki DG, Weise CE, Sarafin HW. A semiquantitative culture method for identifying intravenous-catheter-related infection. N Engl J Med1977; 296:1305-9

15. Ahmed K. Al-Jasser A, Al-Anazi W. Infections caused by non-tuberculous mycobacteria in recipients of hematopoietic stem cell transplantation. Frontiers in Oncology, Novermber 2014.

16. Awadh H, Mansour M, Shorman M. Bacteremia with an unusual pathogen: Mycobacterium neoaurum Case reports in infectious diseases. Volume 2016. 